\title{
Aproximación a la Diabetes Mellitus Oculta en un Servicio de Urgencias Hospitalario
}

\author{
Macarena Bravo Moralesa , Francisco Javier Rodríguez del Río , Ángeles Pérez Jiménezº, \\ A. Alberto León Martín ${ }^{c}$, Patricio Giralt Muiña ${ }^{d}$.
}

\begin{abstract}
${ }^{\text {a }}$ MIR $3^{\circ}$ año de Medicina Familiar y Comunitaria. Centro de Salud Pío XII de Ciudad Real.

${ }^{\mathrm{b}} \mathrm{MIR} 3^{\circ}$ año de Medicina Familiar y Comunitaria. Centro de Salud El Carmen de Ciudad Real.
\end{abstract}

\section{${ }^{c}$ Medicina Familiar y Comunitaria. Hospital General de Ciudad Real.}

\section{d Pediatra. Hospital General de Ciudad Real.}

Correspondencia: Francisco Javier Rodríguez del Río, C/ María Guerrero 17, 13170Miguelturra (Ciudad Real). Telf.: 630823635.

Recibido el 12 de enero de 2009.

Aceptado para su publicación el 29 de enero de 2009.

\section{RESUMEN}

Objetivos. Determinar la prevalencia de diabetes oculta en los pacientes que presentan hiperglucemia en un servicio de urgencias.

Diseño del estudio. Estudio descriptivo transversal (de prevalencia).

Emplazamiento. El marco de atención sanitaria ha sido el servicio de urgencias hospitalario.

Participantes. Pacientes mayores de 20 años que acudieron al Servicio de Urgencias del Hospital General de Ciudad Real durante 2007, y con valores de glucemia plasmática mayor o igual de 140 $\mathrm{mg} / \mathrm{dl}$. El número total de pacientes con glucemia mayor o igual a 140 fue de 4.559 .

Mediciones principales: Variables sociodemográficas y causas de hiperglucemia.

Resultados. La edad media de los pacientes con glucemia mayor o igual a $140 \mathrm{mg} / \mathrm{dl}$ fue de 68,5 años $\pm 13,8$ DE. El $50 \%$ fueron menores de 71 años. La moda en la muestra fue de 75 años. El $52,2 \%$ (2.381) eran hombres. Las causas de hiperglucemia fueron: diabetes mellitus ya conocida $(75,1 \%$ de la muestra, 3.423 pacientes), diabetes mellitus oculta (5,3\% de la muestra, 240 pacientes), hiperglucemia secundaria a otras causas $(19,7 \%$ de la muestra, 896 pacientes). Por lo tanto, la prevalencia de diabetes oculta en nuestra población fue del 5,3\% (IC 95\%: 4,3 - 5,6\%). De los diabéticos conocidos, el 10,3\% eran del tipo 1 (351 pacientes) y el 89,7\% del tipo 2 (3.072 pacientes). Entre las hiperglucemias secundarias, el $18 \%$ lo fueron por tratamiento con corticoides (165 personas), el 32\% por tratamiento oncológico (238 pacientes), el 3\% por algún problema biliar-pancreático (28 pacientes), el $32 \%$ por hiperglucemias aisladas en relación con el estrés del proceso actual (291 pacientes) y el $15 \%$ por intolerancia a la glucosa (131 pacientes).

Conclusiones. La prevalencia de diabetes oculta es parecida a la encontrada en estudios similares. La diabetes oculta se confirma como un problema sanitario de peso epidemiológico (5,3\% de nuestros pacientes) del que podrían derivarse importantes repercusiones sociosanitarias.

Palabras clave. Diabetes Mellitus. Intolerancia a la Glucosa.

\section{ABSTRACT}

Estimation of Hidden Diabetes Mellitus in a Hospital Emergency Department

Objectives. To determine the prevalence of hidden diabetes in patients presenting with hyperglycaemia at a hospital emergency department.

Study design. Cross-sectional descriptive study (prevalence) studio.

Setting. Hospital emergency department.

Participants. Patients over 20 years who attended the Emergency Department of the Hospital General de Ciudad Real during 2007 with plasma glucose levels over $140 \mathrm{mg} / \mathrm{dl}$. The total number of patients with glucose levels higher than $140 \mathrm{mg} / \mathrm{dl}$ was 4,559 .

Main measurements. Socio-demographic variables and cause of hyperglycaemia.

Results. The mean age of patients with glucose levels greater than or equal to $140 \mathrm{mg} / \mathrm{dl}$ was 68.5 years $\pm 13.8 \mathrm{SD}, 50 \%$ were less than 71 years. The mean in the sample was 75 years and $52.2 \%$ (2.381) were men. The causes of hyperglycaemia were: known diabetes mellitus $(75.1 \%$ of the sample, 3,423 patients), hidden diabetes mellitus (5.3\% of the sample, 240 patients), hyperglycaemia secondary to other causes (19.7\% of sample, 896 patients). Therefore, the prevalence of hidden diabetes in our population was $5.3 \%(95 \% \mathrm{Cl}: 4.3-5.6 \%)$. Of those patients with known diabetes, $10.3 \%$ had type 1 (351 patients) and $89.7 \%$ had type 2 (3,072 patients). In $18 \%$ of cases, hyperglycaemia was secondary to corticoid treatment (238 patients), in 3\% to a biliar-pancreatic problem (28 patients), in $32 \%$ to isolated hyperglycaemia related to the stress of their current illness (291 patients) and in $15 \%$ to glucose intolerance (131 patients).

Conclusions. The prevalence of hidden diabetes is comparable to that found in similar studies. Hidden diabetes is confirmed as being a healthcare problem of epidemiological weight (5.3\% of our patients) which could have serious socio-healthcare repercussions.

Key words. Diabetes Mellitus. Glucose Intolerance. 


\section{INTRODUCCIÓN}

La prevalencia de Diabetes Mellitus (DM) en España se sitúa en torno a un 6,5\% para la población entre los 30 y 65 años, oscilando en diferentes estudios entre el 6 y el $12 \%{ }^{1-8}$. Según algunos de estos, la diabetes oculta puede suponer otro $5 \%$, aproximadamen$t^{9}$. Los datos de la Encuesta Nacional de Salud (ENS) muestran cómo, desde 1993 a 2003, la prevalencia de DM ha aumentado del 4,1 al 5,9\%, alcanzando en el rango de edad entre 65 y 74 años el $16,7 \%$, y en los mayores de 75 años el $19,3 \%{ }^{10}$. Según la Encuesta de Salud de Castilla La Mancha, en 200630 el 17,2\% de la población mayor de 65 años declaraba padecer diabetes ${ }^{9}$.

La prevalencia de la diabetes tipo 1 en España se sitúa entre el 0,2-0,3\%, lo que supone entre un 5 y un $10 \%$ del total de personas con diabetes mellitus ${ }^{11}$. La diabetes tipo 2 es la forma predominante. En el 20-50\% se llega al diagnóstico cuando las complicaciones ya están presentes. Del 30 al $50 \%$ de las personas con diabetes ignora su enfermedad. Su prevalencia en España se sitúa en torno al $6 \%$ de la población, aumentando la proporción de forma significativa en relación con la edad y las distintas etnias ${ }^{9}$. Cuanto menor es el nivel socioeconómico, mayor es la prevalencia de DM tipo 2, más frecuentes son los factores de riesgo y peor es el control de la enfermedad, a pesar de una mayor frecuentación en las consultas ${ }^{9}$.

Este aumento de la prevalencia ${ }^{12}$ se puede atribuir a varias causas: por un lado, a la modificación del criterio diagnóstico de $\mathrm{DM}^{13} \mathrm{y}$, por otro, al progresivo envejecimiento de la población, unido a los cambios en los estilos de vida, caracterizados por menor actividad física y hábitos dietéticos que favorecen patologías como la obesidad ${ }^{14}$. Otros factores ${ }^{15}$ son la historia familiar de DM, el sedentarismo, la tolerancia alterada a la glucosa, la hipertensión arterial y la hiperlipemia ${ }^{16}$. El cribado oportunista de la DM en población con factores de riesgo permite detectar los casos de prediabetes y dirigir nuestro trabajo para modificar estilos de vida y prevenir la aparición de $\mathrm{DM}^{17}$.

Los datos disponibles de incidencia de DM tipo 2 en España $^{18}$ oscilan entre 8,1 y 10,8 nuevos casos por 1.000 habitantes-año ${ }^{19}$. La incidencia anual de DM tipo 1, por 100.000 habitantes, oscila entre 9,5 y 25 en menores de 14 años $^{11}$, y se sitúa en 9,9 entre los 15 y 29 años $^{2}$. La incidencia varía según la edad, mostrando dos picos de máxima incidencia: el primero entre los 2 a 5 años y otro en la edad prepuberal. En el grupo de 0 a 14 años no existen diferencias en la incidencia por sexos ${ }^{21}$, mientras que entre 15 y 30 años se observa un claro predominio de varones ${ }^{22}$. En relación con la DM tipo 1 en menores de 16 años, un estudio llevado a cabo en Ciudad Real durante 199921 muestra un incidencia mayor de la esperada, situándose en $26 / 100.000$ casos, sólo superada en un estudio de Castilla León en $2006^{23}$. En cuanto a la prevalencia, se obtienen cifras de alrededor de 2,1/1000 habitantes. La OMS estima que en el año 2002 existían unos 3.000 enfermos (y 190 nuevos casos al año) por cada 100.000 habitantes a nivel mundial. Las previsiones de la OMS para 2030 son de 366 millones de diabéticos, convirtiéndose en una autentica epidemia en los países desarrollados ${ }^{24}$.

La DM esta considerada como uno de los principales problemas de salud pública por el importante coste personal y social para los pacientes que la sufren, así como por los costes sanitarios. En diabéticos tipo 1, en los primeros años de evolución de la enfermedad, los recursos se consumen básicamente en atención extra-hospitalaria (fármacos y material de autoanálisis), siendo las complicaciones agudas frecuentes. Tras este período, los costes se duplican a expensas del tratamiento intrahospitalario de las complicaciones $^{25}$, mayor tasa de reingresos y aumento de la duración de la estancia hospitalaria con respecto a la población no diabética ${ }^{26}$, siendo las complicaciones cardiovasculares las responsables del incremento de la morbilidad hospitalaria ${ }^{27}$ y del $47 \%$ del incremento del $\operatorname{coste}^{28}$. La estancia media de hospitalización aumenta en 3,1 días y la probabilidad de volver a ser hospitalizado un $67 \%$.

En los diabéticos tipo 2 el coste directo medio estimado en el estudio CODE-2 fue de 1.305 euros por paciente y año. De este cómputo total, el $42 \%$ corresponde a gastos de farmacia, el $32 \%$ a costes de hospitalización y el $26 \%$ a gastos de atención ambulatoria ${ }^{29}$. Otros estudios sitúan los costes totales de la DM tipo 2 en 4.378 euros por paciente y año, de los que 2.504 corresponden a costes directos y 1.774 a costes indirectos. En los casos con DM mal controlada los costes son tres veces superiores, multiplicándose, respectivamente, por 3, 5 ó 10 en presencia de complicaciones microvasculares, macrovasculares o ambas complicaciones crónicas.

Con respecto a la mortalidad de DM, en la mayoría de los países desarrollados supone, entre las causas de defunción, del cuarto al octavo lugar, representando la tercera causa de mortalidad en mujeres y la séptima en hombres. Según el estudio "Mortalidad por diabetes mellitus. España y Castilla La Mancha 19802004", se produce una media de 8.857 defunciones anuales por DM, lo que supone el 2,7 \% de defunciones con respecto al total de causas de muerte $(1,8 \%$ en varones y $3,6 \%$ en mujeres). La tasa bruta de mortalidad por diabetes ha aumentado en España, entre 1980 y 2004 , en un $24,37 \%$, siendo el incremento 
mayor en hombres $(42,5 \%)$ que en mujeres $(15,11 \%)$. Sin embargo, la tasa ajustada de mortalidad por la misma causa ha descendido en España en este periodo $(-19,39 \%)$. En Castilla-La Mancha la DM es causa del $2,9 \%$ de las defunciones con respecto al total de éstas, con una media anual de 468 muertes30. Con respecto a otras Comunidades Autónomas, CastillaLa Mancha ocupa la octava posición en número de defunciones por DM entre 1999 y 200430. Durante el periodo 1980-2004, en Castilla-La Mancha, la mayor tasa ajustada de mortalidad por diabetes correspondió a Ciudad Real, seguida de Albacete, y la menor a Guadalajara $^{30}$.

El objetivo del estudio ha sido determinar la prevalencia de diabetes mellitus oculta y de intolerancia a hidratos de carbono en las personas que presentan hiperglucemia en un servicio de Urgencias.

\section{MATERIAL Y MÉTODO}

La muestra procede de los pacientes que fueron atendidos en el Servicio de Urgencias del Hospital General de Ciudad Real en el periodo de un año (11/01/07 a

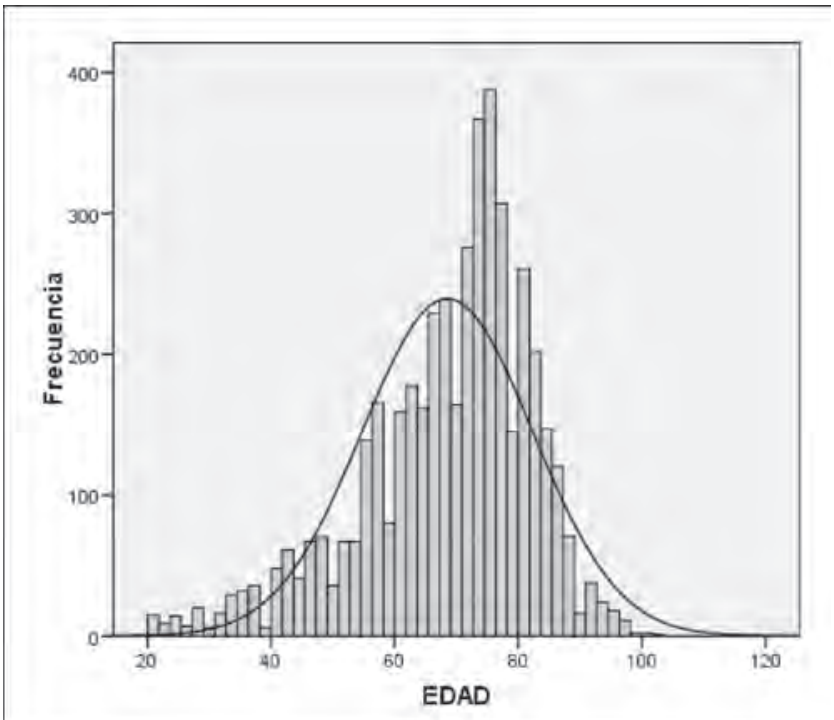

Figura 1. Distribución de los pacientes según su edad.

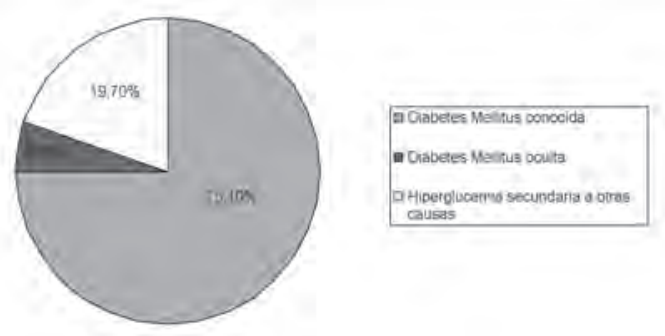

Figura 2. Tipos de Hiperglucemias.

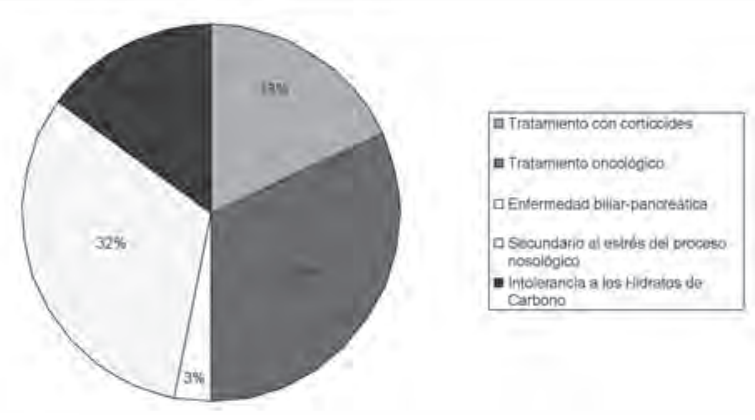

Figura 3. Tipos de Hiperglucemia secundaria.

11/01/08). Los criterios de inclusión fueron: pacientes mayores de 20 años que acudieron al Servicio de Urgencias, previamente no diagnosticados de diabetes y con glucemia al azar mayor o igual a $140 \mathrm{mg} / \mathrm{dl}$. Los criterios de exclusión fueron: menores de 20 años, glucemia al azar menor o igual a $139 \mathrm{mg} / \mathrm{dl}$, enfermedades (diabetes mellitus ya conocida, SIDA, otras enfermedades concomitantes como hepatopatía crónica, insuficiencia renal crónica, hipertiroidismo, síndrome de Cushing o acromegalia, y procesos intercurrentes como fiebre, gastroenteritis o infecciones urinarias, respiratorias, bucales, etc.), pacientes politraumatizados y pacientes en tratamiento con diuréticos, betabloqueantes, betaadrenérgicos, corticoides, antimicrobianos (sulfamidas, rifampicina, pentamidina), fibratos, análogos de somatostatina y hormona del crecimiento, anticonceptivos orales o analgésicos a altas dosis (salicilatos, paracetamol).

En los pacientes atendidos que cumplieron los criterios de inclusión se estudiaron, a través de la historia clínica, sus hábitos higiénico-dietéticos, los antecedentes familiares y personales y la valoración analítica realizada.

El diseño corresponde a un estudio epidemiológico observacional y descriptivo, de carácter transversal. Se pretende estudiar la relación entre cifras de glucemia halladas al azar en el servicio de Urgencias hospitalario y el diagnóstico de Diabetes Mellitus oculta, verificado posteriormente mediante determinación analítica.

Para estudiar la prevalencia de DM oculta, se analizó la base de datos de los registros informáticos hospitalarios y de atención primaria de los pacientes que acudieron al Servicio de Urgencias Hospitalario de Ciudad Real durante el periodo indicado. A aquellos pacientes con una glucemia mayor o igual de 140 (según el protocolo de la Asociación Americana de Diabetes, ADA) (tabla 1) y con criterios de inclusión, se procedió a realizar una sobrecarga oral de glucosa (SOG) de forma ambulatoria (cuando no se realizó, se contactó con su centro de salud para completar el 
estudio). Si el resultado fue positivo se calificó de diabetes mellitus oculta y, si el resultado fue negativo, de intolerancia a los hidratos de carbono, si se cumplían los criterios de ADA.

La intolerancia a los hidratos de carbono (IHC) se diagnostica mediante sobrecarga oral de 75 gramos de glucosa: glucemia basal en plasma venoso inferior a 126 mg/dl y glucemia a las 2 horas de la SOG mayor o igual a 140 e inferior a 199 mg/dl. Realización de la SOG: consiste en administrar 75 mg de glucosa en $250 \mathrm{ml}$ de agua a primera hora de la mañana, tras 10-12 horas de ayuno. Se realiza extracción de sangre venosa a las 2 horas. Previamente se debe hacer una dieta normocalórica con un aporte superior a 150 gramos de hidratos de carbono las 48-72 horas anteriores y se debe mantener una actividad física normal las 48-72 horas previas. La prueba debe ser realizada sólo en sujetos ambulantes y nunca en pacientes encamados $u$ hospitalizados. El paciente ha de permanecer en reposo y sin fumar durante todo el tiempo que dura la prueba. No debe estar recibiendo medicación que pueda alterar la tolerancia a la glucosa, por lo que se recomienda suspender la medicación una semana antes. Si en los días previos a la prueba el paciente hubiera atravesado una situación de estrés (IAM, infección, traumatismo grave, etc.), se debe dejar pasar algún tiempo (8-12 semanas) antes de someterlo a la prueba.

\section{RESULTADOS}

El número total de pacientes con glucemia mayor o igual a $140 \mathrm{mg} / \mathrm{dl}$ fue de 4.559 personas y la edad media de 68,5 años $\pm 13,8$ DE (rango de edad entre 20 y 103 años). La distribución por edad de los pacientes estudiados sigue una distribución normal o de Gauss (figura 1). El 50\% tiene una edad menor de 71 años (mediana 71 años). La moda o edad más prevalente en la nuestra es 75 años. En relación al sexo de los pacientes, el 52,2\% (2.381 personas) eran hombres y el 47,8\% (2.178 personas) mujeres.

La prevalencia de diabetes mellitas oculta en los pacientes con glucemia mayor o igual a $140 \mathrm{mg} / \mathrm{dl}$ al azar fue del 5,3\% del total (IC 95\%: 4,3 - 5,6\%) (240 personas entre 4.559 pacientes con glucemia mayor o igual a 140mg/dl). En 131 pacientes se identificó intolerancia a los hidratos de carbono (2,9\%). De los 240 pacientes con diabetes mellitus oculta, 124 $(51,7 \%)$ eran hombres y $116(48,3 \%)$ eran mujeres. La edad donde la diabetes mellitus oculta resultó más frecuente (92,4\% de 240 pacientes), fue en mayores de 45 años. De los 131 pacientes con intolerancia a los hidratos de carbono, 76 (58\%) eran hombres y 55 (42\%) eran mujeres. La edad donde la intolerancia a los hidratos de carbono resultó más frecuente (87,9\% de 131 pacientes) también fue en mayores de 45 años.

Además del análisis de las 4559 historias clínicas, en el estudio de la frecuentación hospitalaria (incluyendo a los pacientes que no cumplieron los criterios de inclusión) se consiguieron los siguientes resultados:

- En relación al tipo de hiperglucemia posible, se encontró (figura 2): diabetes previamente conocida en el $75,1 \%$ de la muestra (3423 pacientes), diabetes mellitus oculta en el 5,3\% (240 pacientes) e hiperglucemia secundaria a otras causas en el 19,7\% (896 pacientes).

- En relación al tipo de diabéticos que frecuentan las urgencias hospitalarias, se obtuvo: 351 diabéticos tipo 1 (10,3\% de los diabéticos conocidos) y 3.072 diabéticos tipo 2 (89,7\% de los diabéticos conocidos).

- En relación al tipo de hiperglucemia secundaria (figura 3), se obtuvo: 165 pacientes con hiperglucemia secundaria a tratamiento con corticoides (18\% de las hiperglucemias secundarias y 3,5\% de los 4559 pacientes), 283 pacientes con hiperglucemia secundaria a tratamiento oncológico (32\% de las hiperglu-

\begin{tabular}{|c|c|c|c|}
\hline & $\begin{array}{c}\text { Glucemia basal } \\
\text { (mg/dl) }\end{array}$ & $\begin{array}{l}\text { Glucemia azar } \\
\text { (mg/dl) }\end{array}$ & $\begin{array}{l}\text { Glucemia } 2 \text { h } \\
\text { SOG (mg/dl) }\end{array}$ \\
\hline Normal & $\begin{array}{l}<110 \text { (OMS) o bien } \\
\quad<100 \text { (ADA) y }\end{array}$ & -- & $<140$ \\
\hline $\begin{array}{r}\text { Prediabetes } \\
\text { - TAG }\end{array}$ & --- & --- & $140-199$ \\
\hline - GBA & $\begin{array}{l}>110 \text { (OMS) o bien } \\
100 \text { (ADA) y < 126 }\end{array}$ & --- & -- \\
\hline Diabetes & $>126$ ó & $\begin{array}{l}>200 \text { mas } \\
\text { síntomas ó }\end{array}$ & $>200$ \\
\hline
\end{tabular}

Tabla 1. Criterios diagnósticos. Las cifras se refieren a glucemia en plasma venoso (mg/dl). Los valores a las 2 horas son tras SOG 75 gramos. 
cemias secundarias y 6,3\% de los 4559 pacientes), 28 pacientes con hiperglucemia secundaria a algún problema biliar-pancreático ( $3 \%$ de las hiperglucemias secundarias y 0,6\% de los 4559 pacientes), 291 pacientes con hiperglucemia aisladas en relación con el estrés del proceso patológico (infecciones, fiebre y otros procesos intercurrentes) por el que acudieron a urgencias $(32 \%$ de las hiperglucemias secundarias y $6,3 \%$ de los 4559 pacientes) y 131 pacientes que tras su estudio presentaron intolerancia a los hidratos de carbono, previamente no conocida (15\% dentro de este grupo y el $3 \%$ de todos los pacientes).

\section{DISCUSIÓN}

Nuestro estudio recoge información de la población mayor de 20 años de la provincia de Ciudad Real (Castilla-La Mancha) que acudió a urgencias hospitalarias a lo largo del año 2007. Observamos durante este periodo a los pacientes que presentaron en las analíticas glucemias mayor o igual a 140 mg/dl, cuántos eran diabéticos y no lo sabían, cuántos de ellos estaban en una situación de "prediabetes", y al mismo tiempo describimos cuántos eran diabéticos tipo 1 o 2, pudiendo obtener información de la frecuentación hospitalaria y un cálculo aproximado del peso sobre el gasto sanitario que suponen estos pacientes (el $75,1 \%$ de los pacientes eran diabéticos conocidos, 351 pacientes eran DM tipo 1 y 3.072 pacientes eran DM tipo 2). También describimos la carga asistencial que suponen las hiperglucemias secundarias a otros procesos, las cuales, a nivel de los pacientes que acude a urgencias hospitalarias de Ciudad Real, suponen el $19,7 \%$ de los que presentan en control analítico glucemia mayor o igual a $140 \mathrm{mg} / \mathrm{dl}$.

Este estudio presenta la ventaja de no plantear problemas éticos. Su duración fue limitada y su reproductibilidad es sencilla, siendo apropiado para el estudio de enfermedades crónicas como la diabetes. Sin embargo, también presenta inconvenientes, como la imposibilidad de conocer la secuencia temporal, dado que la información sobre los factores de riesgo y la enfermedad se recogen a la vez.

El número total de pacientes estudiados fue de 4.559 pacientes, los cuales presentaron glucemia al azar mayor o igual a 140 mg/dl. El número total de pacientes que acudieron a urgencias hospitalarias, con edad mayor de 20 años, durante el año 2007 fue de 71.666 pacientes. De ellos cumplieron criterios de diabetes mellitus oculta e intolerancia hidrocarbonada un total de 371 pacientes. Consideramos que se trata de una muestra suficiente y adecuada, tratándose de un estudio realizado en pacientes que cumplen los criterios de inclusión previamente determinados, atendidos en el Servicio de Urgencias del Hospital General de Ciudad Real, el cual abarca la población de Ciudad
Real. La muestra estudiada es muy similar en su composición por edad y sexo a la población de referencia (población de Castilla-La Mancha30), por lo que podemos considerarla representativa de la misma. La prueba diagnostica utilizada fue la SOG, a diferencia de la mayoría de los estudios realizados en España2-4, en los cuales se realiza la determinación de glucosa en sangre capilar.

En España existen pocos estudios poblacionales que abarquen grandes grupos de población, como provincias o comunidades autónomas, salvo los estudios de Aragón ${ }^{5}$ y Cataluña ${ }^{2}$. El análisis sobre grandes poblaciones dificulta y alarga el trabajo de campo, pero concede mayor validez al estudio.

En cuanto al método diagnóstico, el comité de expertos de la OMS en su último informe de 199912, y recientemente el Grupo Europeo de la Federación Internacional de Diabetes en su Guía para la Diabetes Mellitus tipo 2, asumen el punto de corte de la glucemia basal propuesto dos años antes por la Asociación Americana de Diabetes de $126 \mathrm{mg} / \mathrm{dl}^{11}$, pero mantienen la recomendación de realizar SOG a aquellas personas con glucemia basal entre 110-125 mg/dl, que son los criterios diagnósticos actuales que se deben de aplicar en España. La realización de extracción venosa permite aumentar la validez y fiabilidad de los análisis, determinando la glucosa en laboratorio, lo que disminuye la variabilidad de este parámetro con respecto al uso de reflectómetros para la determinación de glucemia capilar.

Los resultados muestran que el $5,3 \%$ de los pacientes estudiados presentan diabetes mellitus oculta, resultados similares a los aportados en el año 2003 por el Ministerio de Sanidad y Consumo, que describía una prevalencia del $5 \%$ de diabetes mellitus oculta en el total de la población9. La distribución es similar en ambos sexos (51,7\% hombres y $48,3 \%$ mujeres), siendo más frecuente en los pacientes mayores de 45 años, de forma similar a los datos reflejados en el Plan Integral de Diabetes Mellitus de Castilla-La Mancha $^{30}$.

El estudio norteamericano NHANES III, sobre población entre 40-74 años, ofrece cifras de prevalencia de intolerancia a los hidratos de carbono de $15,8 \%$, y en el estudio Asturias ${ }^{8}$, para el mismo rango de edad, se encuentra una prevalencia similar de ITG del 16\%. En EE.UU. la diabetes es considerada actualmente uno de los principales problemas de salud pública por su elevada y creciente prevalencia y por el elevado coste sanitario que provoca. En España estamos bastante cerca de las tasas norteamericanas y probablemente asistamos en los próximos años a un incremento progresivo del impacto de esta enfermedad y de sus complicaciones en nuestra población. En los resulta- 
dos obtenidos en este trabajo, la prevalencia de intolerancia a los hidratos de carbono, en el total de pacientes atendidos en urgencias con glucemia al azar mayor o igual a $140 \mathrm{mg} / \mathrm{dl}$, es del 3\%. Las ITG, dentro de las hiperglucemias secundarias (todos aquellos que no cumplen los criterios de diabetes tipo 1, tipo 2 , ni diabetes oculta), representan el 15\%. La diferencia en el resultado entre los estudios previamente citados y el presente trabajo se debe al dintel glucémico utilizado (a partir de $140 \mathrm{mg} / \mathrm{dl}$ ), a que muchos pacientes no cumplieron criterios de inclusión por padecer procesos intercurrentes y a que no se estudiaron posteriormente para comprobar si tras la resolución del proceso se trataba de una ITG, una diabetes mellitus oculta o bien una hiperglucemia ocasional secundaria a la situación estresante. Otra posibilidad que barajamos es que la población que acude a urgencias presenta procesos normalmente sintomáticos, como poliuria, polidipsia y polifagia, entre otras posibilidades clínicas, y sin embargo las ITG son procesos clínicamente asintomáticos. En cuanto a la distribución de ITG por sexos, se observa en nuestro estudio una distribución parecida en ambos sexos $(58 \%$ hombres y $42 \%$ mujeres). La ITG es más prevalente en los mayores de 45 años, que igualmente es superponle a lo descrito en el Plan Integral de Diabetes Mellitus de Castilla-La Mancha ${ }^{30}$.

\section{BIBLIOGRAFÍA}

1. Goday A, Díaz Cadórniga F, Delgado E, Soto E, Vázquez JA, De Pablos P. Epidemiología de la diabetes mellitus. Endocrinol Nutr 2002; 49:113-26.

2. Castell C, Tresserras R, Serra J, Goday A, Lloveras G, Salleras LI. Prevalence of Diabetes in Catalonia (Spain): an oral glucose tolerance test based population study. Diabetes Res Clin Pract 1999; 43:33-40.

3. Franch Nadal J, Álvarez Torrices J C, Álvarez Guisasola F, Diego Domínguez F, Hernández Mejía R, Cueto Espinar A. Epidemiología de la Diabetes Mellitus en la provincia de León. Med Clin (Barc) 1992; 98:607-11.

4. Bayo J, Sola C, García F, Latorre PM, Vázquez JA. Prevalencia de la diabetes mellitas no dependiente de la insulina en Lejona (Vizcaya). Med Clin (Barc) 1993; 101:609-12.

5. Tamayo-Marco B, Faure-Nogueras E, Roche-Asensio MJ, Rubio-Calvo E, Sánchez-Oriz E, Salvador-Oliván JA. Prevalence of diabetes mellitus and impaired glucose tolerante in Aragon, Spain. Diabetes Care 1997; 20:534-6.

6. De Pablos-Velasco PL, Martínez-Martín FJ, Rodríguez-Pérez F, Anía BJ, Losada A, Betancor P. Guía Study. Prevalence and determinants of diabetes mellitus and glucosa intolerance in canarian caucasian population comparison of the ADA and the 1985 WHO criteria: The guia study. Diabet Med 2001; 18:235-41.

7. Cerezo J, Tormo MJ, Rodríguez-Barranco M, Navarro C, Marco Aledo R, Egea Caparrós JM, et al. Diabetes y obesidad en la población adulta de la Región de Murcia. Consejería de Sanidad de la Región de Murcia. Serie Informes, n 40, 2005.

8. Botas Cervero P, Delgado Alvarez E, Castaño Fernandez G. Prevalencia de diabetes mellitus e intolerancia a la glucosa en población entre 30 y 75 años en Asturias, España. Rev Clin Esp 2002; 202:421-7.

9. Encuesta Nacional de Salud, 2003. Ministerio de Sanidad y Consumo. Disponible en http://www.msc.es/estadEstudios/ estadisticas/docs/ENSE2003_SN.pdf.

10. Ministerio de sanidad y consumo. Secretaría General de Sanidad. Estudio sobre la situación de la diabetes en España. Madrid; 2004.
11. American Diabetes Association. Expert Committee on the Diagnosis and Classification of Diabetes Mellitus. Report of the Expert Committee on the diagnosis and classification of diabetes mellitus. Diabetes Care 1997; 20:1183-97.

12. World Health Organization: Definition, diagnosis and classification of diabetes mellitus and its complications: report of a WHO Consultation, Part 1. Diagnosis and classification of diabetes mellitus. Geneve: World Health Organization; 1999.

13. Informe anual del Sistema Nacional de Salud 2004. Ministerio de Sanidad y Consumo. Disponible en: http://www.msc.es/organizacion/sns/informeAnualSNS/informe2004.htm.

14. Shaw JT, Purdie DM, Neil HA, Levy JC, Turner RC. The relative risks of hyperglycaemia, obesity and dyslipidaemia in the relative of patients with type 2 diabetes mellitus. Diabetología 1999; 42:24-7.

15. Haffner SM. Epidemiology of type 2 diabetes: risk factors. Diabetes Care 1998; 2I:C3-C6.

16. Tuomilehto J, Lindström J, Eriksson JG, Valle TT, Hamalainen $\mathrm{H}$, Hanne-Parikka $\mathrm{P}$, et al. Prevention of type 2 diabetes mellitus by changes in lifestyle among subjects with impaired glucose tolerance. N Engl J Med 2001; 344:1343-50.

17. Vázquez JA, Gaztambide S, Soto-Pedre E. Estudio prospectivo a 10 años sobre la incidencia y factores de riesgo de la diabetes mellitus tipo 2. Med Clin 2000; 115:534-9.

18. Valdés Hernández S, Botas Cervero P, Delgado Álvarez E, Díaz Cadórniga FJ, Álvarez Menéndez F. Incidencia de diabetes tipo 2 en la Comunidad Asturiana. Av Diabetol 2006; 22:[Abstract].

19. Goday A, Serrano-Ríos M, Castell C, Lloveras G, Gutiérrez R, Martull $P$, et al. Los estudios de incidencia de diabetes mellitus tipo 1 en España. Análisis comparativo y consenso de metodología estandarizada. Av Diabetol 1996; 12:24-8.

20. Giralt Muiña P, Santillana Ferrer L, Madrigal Barchino D, Merlo Garrido A, Toledo de la Torre B, Anaya Barea F. Incidencia en menores de 16 años y prevalencia de la diabetes mellitus tipo 1 en la provincia de Ciudad Real. Ann Esp Pediatr 2001; 55:213-8.

21. Carrillo Domínguez A. Incidencia de la diabetes mellitus tipo 1 en las Islas Canarias (1995-1996). Rev Clin Esp 2000; 200:25760.

22. Bahíllo Curieses MP, Hermoso López F, García Fernández JA, Ochoa Sangrador C, Rdrigo Palacios J, De la Torre Santos SI. Epidemiología de la diabetes tipo 1 en menores de 15 años en las provincias de Castilla León. Rev. Anales de Pediatría 2006; 65:15-21.

23. Wild S, Roglic G, Green A, Sicree R, King H. Global prevalence of Diabetes. Estimates for the 2000 year and projections for 2030. Diabetes Care 2004; 27:1047-53.

24. Pascual JM, González C, De Juan S, Sánchez C, Sánchez B, Pérez M. Impact of diabetes mellitus on hospitalization costs. Med Clin (Barc) 1996; 107:207-10.

25. Carral F, Olveira G, Salas J, García L, Sillero A, Aguilar M. Care resource utilization and direct cost incurred by people with diabetes in a spanish hospital. Diabetes Res Clin Pract 2002; 56:27-34.

26. Carral F, Aguilar M, Olveira G, Mangas A, Domenech I, Torres I. Increased hospital expenditures in diabetic patients hospitalized for cardiovascular diseases. J Diabetes Complications 2003; 17:331-6.

27. Olveira-Fuster G, Olvera-Márquez P, Carral-Sanlaureano F, González-Romero S, Aguilar-Diosdado M, Soriguer-Escofet F. Excess hospitalizations, hospital days and inpatient costs among people with diabetes in Andalusia (Spain). Diabetes Care 2004; 27:1904-9.

28. Mata M, Antoñanzas F, Tafalla M, Sanz P. El coste de la diabetes tipo 2 en España. El estudio CODE-2. Gac Sanit 2002; 16:51120.

29. Ballesta M, Carral F. Costes directos e indirectos de la diabetes mellitus tipo 2. Av Diabetol 2002; 18:2).

30. Consejería de Sanidad de Castilla-La Mancha. Plan Integral de diabetes de Castilla La Mancha 2007-2010. Toledo: Junta de Comunidades de Castilla-La Mancha; 2007. 